\title{
A Metamaterial for Superscattering Light
}

\section{A team has engineered a subwavelength structure that features a greatly enhanced capacity to scatter microwave light.}

\section{by Yongmin Liut}

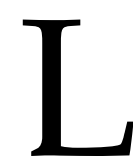

ight scattering is a common optical phenomenon, much like reflection, refraction, and absorption. Small molecules in the atmosphere scatter sunlight more efficiently at the shorter wavelengths of the solar spectrum, giving the sky its blue color on a clear day. And larger vapor droplets scatter light over the entire visible spectrum, lending clouds their white appearance. In general, the intensity and direction of the scattered light depend on its wavelength and on the characteristics of the scatterer-such as size, geometry, and refractive index. Researchers have tuned these characteristics to engineer structures that scatter light in specific-and often surprising-ways. Schemes for diminished scattering, or "cloaking," have been proposed or realized [1, 2], as have schemes that selectively enhance scattering in a desired direction [3-5]. Now, a team led by Hongsheng Chen at Zhejiang University, China, and Baile Zhang at Nanyang Technological

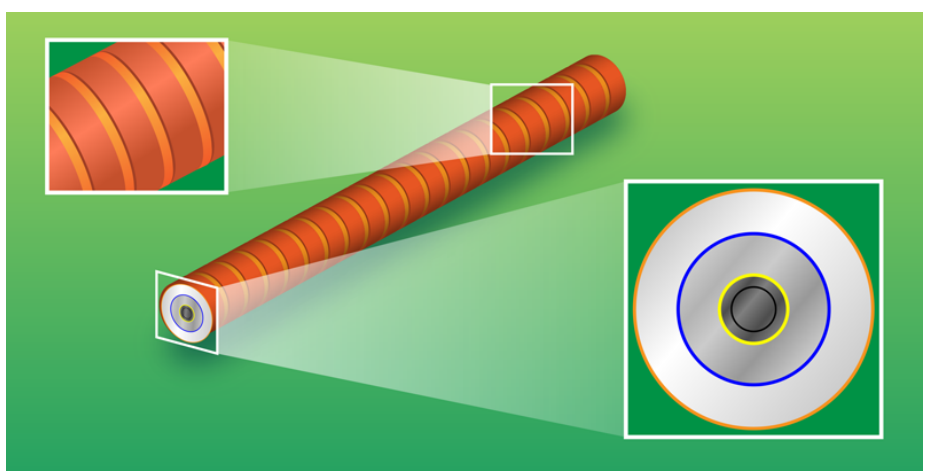

Figure 1: Sketch of the superscatterer engineered by Chen, Zhang, and co-workers-a cylinder made of three concentric cylindrical metasurfaces separated by dielectric materials. (APS/Alan Stonebraker)

$\dagger$ Departments of Mechanical and Industrial Engineering and Electrical and Computer Engineering, Northeastern University, Boston, MA, USA
University, Singapore, has designed a subwavelength-sized structure whose ability to scatter light is greatly enhanced, providing the first experimental demonstration of the socalled superscattering effect [6]. This phenomenon could be harnessed for a variety of applications, from boosting the efficiency of antennas and energy-harvesting devices to improving the resolution of imaging schemes.

At the microscopic level, light scattering is the process by which an incident light wave perturbs the electrons in an object, inducing dipole moment oscillations that emit radiation. The scattering strength can be measured by the scattering cross section, defined as the ratio of the scattered power to the intensity of the incident light wave. For a subwavelength scatterer-a scatterer that is very small compared to the wavelength, like an atom in visible light-it has been rigorously proven that the maximum scattering cross section is $3 \lambda^{2} / 2 \pi$ and $2 \lambda / \pi$ in $3 D$ and $2 D$, respectively, where $\lambda$ is the wavelength of the scattered light [7]. This "singlechannel" limit assumes that the radiation is emitted solely via electric dipole transitions. The limit has been shown to apply to almost all subwavelength scatterers studied in the past. But in a seminal theoretical paper from 2010, researchers proposed how to overcome this fundamental limit by introducing additional modes-oscillations of higherorder electric moments, such as quadrupole, hexapole, and the like-each with the same resonant frequency [8]. If those modes act as multiple channels that contribute simultaneously and constructively to the scattering process, one could drastically enlarge the scattering cross section of a subwavelength scatterer. The design in the 2010 proposal relied on the multiple optical plasmon modes of a multilayered nanorod [8]. (These plasmon modes are collective excitations of the electrons at the material's surface.) However, an experimental demonstration of this concept has been impeded by two problems: the finite conductivity of the metal in the optical region of the spectrum leads to loss of scattering power and fabricating the nanostructure is complex.

Chen, Zhang, and their colleagues have overcome these issues and demonstrated a superscattering effect at two frequencies in the microwave region [6]. Guided by analytical and numerical calculations, they designed a superscatterer made of a cylinder with a diameter of $35.96 \mathrm{~mm}$, consist- 


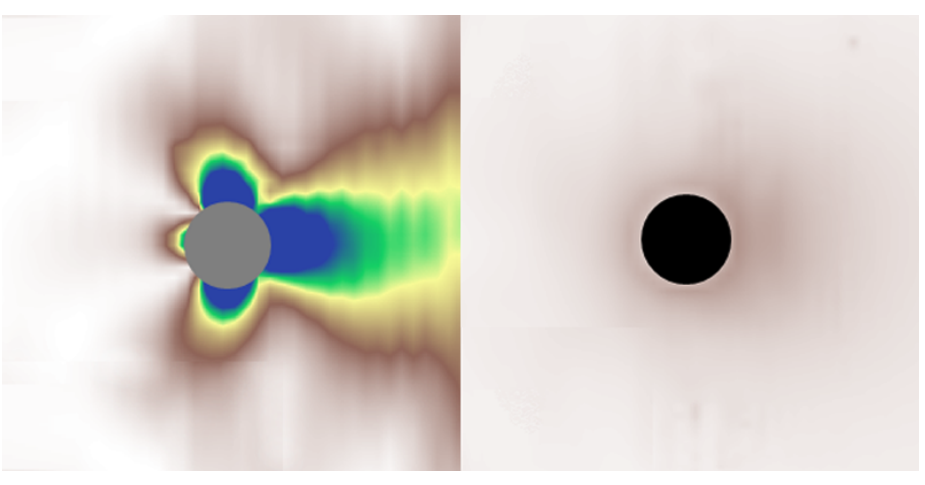

Figure 2: Scattering pattern observed for the superscatterer (left) and for a control sample with the same geometry but containing no metasurfaces (right). (C. Qian et al. [8])

ing of three concentric cylindrical metasurfaces separated by dielectric materials. Each metasurface is composed of periodic copper strips whose periodicity and thickness are much smaller than the wavelength (Fig. 1). Based on the so-called effective media theory, the metasurface functions as an ultrathin slab with an extremely large refractive index that confines surface waves with microwave frequencies. These waves are transverse electric (TE) waves, meaning that their electric field is polarized along the axis of the rod. By carefully tailoring the geometry of the metasurfaces via the periodicity and width of the copper strips, the metasurface can be made to support multiple surface TE modes that are degenerate; that is, they all resonate at the same frequency. The superscatterer designed by the authors supports resonating dipolar and quadrupolar modes at both working frequencies of 2.2 and $3.73 \mathrm{GHz}$. What's more, the surface conductivity of the superscatterer leads to very low dissipated power-overcoming the losses that were a major hurdle in prior work.

Systematic measurements of the scattered waves unambiguously demonstrated the superscattering effect in both the near-field and far-field region around the structure. Such measurements allowed the authors to obtain the most important characteristics of the scattering process, including the local field distribution in the near field as well as the scattering cross section and the scattering directionality. The measured total scattering cross sections at 2.2 and $3.73 \mathrm{GHz}$ exceeded by four times the single-channel limit, agreeing very well with the authors' calculations. In addition to the cross-section enhancement, the authors also observed that the radiation pattern of the scatterer was directional, with most of the scattering intensity going in the forward direction. Both the directionality and the scattering boost were confirmed by comparing the superscatterer with a control sample-a homogeneous copper rod with the same geometry as the superscatterer but with no metasurfaces (Fig. 2).

The work by Chen, Zhang, and colleagues demonstrates that we can substantially enhance the light-matter interaction on the subwavelength scale by engineering the resonant modes of a scatterer. The result opens up exciting opportunities for both fundamental studies and practical applications. At the fundamental level, it would be intriguing to study the combination of superscattering effects with wave propagation phenomena in random media. Such media, which consist of many randomly distributed scatterers, exhibit interesting phenomena like Anderson localization, coherent backscattering, and memory effects. Novel phenomena could arise in random media in which the scatterers are superscatterers. In terms of applications, one promising device is a superscatterer-based antenna with high efficiency and superior directionality for wireless communication, data transmission, and remote sensing. We already have the technology to design proper metasurfaces for these applications. Finally, future research may expand the application of the superscattering effect beyond the microwave frequency, which could have important implications in the areas of imaging, sensing, optical displays, and energy harvesting. While challenging, this seems possible at least at infrared wavelengths, given some recent demonstrations of low-loss materials and structures suitable for such wavelengths, such as hexagonal boron nitride [9] and dielectric-gold core-shell microparticles [10].

This research is published in Physical Review Letters.

\section{REFERENCES}

[1] A. Alù and N. Engheta, "Achieving transparency with plasmonic and metamaterial coatings," Phys. Rev. E 72, 016623 (2005).

[2] B. Edwards, A. Alù, M. G. Silveirinha, and N. Engheta, "Experimental verification of plasmonic cloaking at microwave frequencies with metamaterials," Phys. Rev. Lett. 103, 153901 (2009).

[3] S. Person, M. Jain, Z. Lapin, J. J. Sáenz, G. Wicks, and L. Novotny, "Demonstration of zero optical backscattering from single nanoparticles," Nano Lett. 13, 1806 (2013).

[4] Y. H. Fu, A. I. Kuznetsov, A. E. Miroshnichenko, Y. F. Yu, and B. Luk'yanchuk, "Directional visible light scattering by silicon nanoparticles," Nat. Commun. 4, 1527 (2013).

[5] K. Yao and Y. Liu, "Controlling electric and magnetic resonances for ultra-compact nanoantennas with tunable directionality," ACS Photonics 3, 953 (2016).

[6] C. Qian, X. Lin, Y. Yang, X. Xiong, H. Wang, E. Li, I. Kaminer, B. Zhang, and $\mathrm{H}$. Chen, "Experimental observation of superscattering," Phys. Rev. Lett. 122, 063901 (2019).

[7] C. J. Foot, Atomic Physics, Vol. 7 of Oxford Master Series in Physics (Oxford University Press, New York, 2005).

[8] Z. Ruan and S. Fan, "Superscattering of light from subwavelength nanostructures," Phys. Rev. Lett. 105, 013901 (2010).

[9] C. Qian, X. Lin, Y. Yang, F. Gao, Y. Shen, and J. Lopez, "Multifrequency superscattering from subwavelength hyperbolic structures," ACS Photonics 5, 1506 (2018). 


\section{Phys̄īcs}

[10] K. Yao and Y. Liu, "Infrared plasmonic resonators based on

10.1103/Physics. 12.14 (2017). 